\title{
JRRD
}

Volume 49, Number 9, 2012

Pages 1321-1330

http://dx.doi.org/10.1682/JRRD.2011.11.0221

\section{Transtibial prosthetic suspension: Less pistoning versus easy donning and doffing}

Hossein Gholizadeh, MEngSc;1* Noor Azuan Abu Osman, PhD;1 Arezoo Eshraghi, MSc;1 Sadeeq Ali, BSc;1

Stefan Karl Sævarsson, MSc;2 Wan Abu Bakar Wan Abas, PhD;1 Gholam Hossein Pirouzi, BSc1

1Department of Biomedical Engineering, Faculty of Engineering, University of Malaya, Kuala Lumpur, Malaysia;

2Department of Biomedical Engineering, University of Calgary, Calgary, Canada

\section{INTRODUCTION}

The main roles of the suspension systems incorporated

into lower-limb prostheses are to hold the prosthesis on the residual limb and to decrease the motion that takes place at the bone-skin-liner-socket interface during ambulation (pistoning, vertical movements within the socket) [1]. Effective suspension systems and prosthetic components can improve a person with amputation's gait and decrease his or her energy expenditure [2-3]. Prosthetic limbs should have an intimate fit with the residual limb in order to replace the lost body part with a device that offers high levels of comfort and satisfaction [3-6]. Individuals with amputation believe that both the suspension method and the fitting of a prosthetic device 
have significant effects on their overall satisfaction with

the prosthesis [6-8]. Several questionnaires have been

developed and a number of prosthetics surveys have been

conducted to analyze patient satisfaction with prosthetic

devices. The majority of researchers prefer the Prosthesis

Evaluation Questionnaire (PEQ) as a means of evaluating

differences in function, performance, and satisfaction

between the different components or techniques of prosthetics

fabrication and adjustment (Appendix, available

online only) Good reliability and validity have been

reported for the PEQ [9-11].

Evidence shows that silicone liners are preferred by

many people with lower-limb amputation because they offer enhanced suspension and fit within the socket as well as improved function [3,7-8,12]. Previous research

on the silicone liners has found that patient comfort and

satisfaction are particularly higher in contrast with other

suspension systems, such as the belt for patellar tendon

bearing socket $[3,8,12]$. Silicone liners are believed to be

more effective in controlling the pistoning within the

prosthetic socket than polyethylene foam (pelite) liners.

Pistoning at the socket-liner interface is said to be lower

with silicone liners $(1-5 \mathrm{~mm})$ than with pelite liners $(6.0$

$41.7 \mathrm{~mm}$ ) [13-21].

Based on the literature, the pistoning is correlated with

the prosthetic suspension system and fit [15]. Thus, both

clinicians and researchers should be able to determine the

quality of suspension and prevent the negative effects of

pistoning (such as gait deviation, skin breakdown, and discomfort)

by pistoning measurement [13-22].

A number of methods exist to measure the pistoning

of various interfaces within the socket (liner-socket) or the

residual limb (bone-soft tissue). These include X-ray

[12,20,23-25], spiral computerized tomography [26], and

photoelectric sensors [22]. These measurement methods 
are mostly useful for measuring the bone movement

inside the socket. Recently, two new methods were introduced

for the liner-socket interface in transtibial prostheses:

a photographic method and a motion analysis system

[16-19]. The literature review revealed that the majority

of researchers measured the pistoning during quiet standing

(static) and only a few had evaluated the pistoning that

occurred inside the socket during gait (dynamic) [15].

A previous study by Gholizadeh et al. revealed low

levels of pistoning for the seal-in suspension (Seal-In X5

liner, Össur; Reykjavik, Iceland) than the locking system

(Dermo liner, Össur) during standing [16]. The findings

of that study motivated this current research and

prompted investigation on the effects of these suspension

systems during gait along with patient satisfaction. To

our knowledge, no study has previously compared the

quality of suspension systems during gait and the associated

levels of patient satisfaction.

\section{METHODS}

\section{Subjects}

Ten subjects with unilateral transtibial amputation participated in this study. We determined the participants' mobility grade based on the guidelines of the American Academy of Orthotists \& Prosthetists [27]. Table 1 lists subject characteristics.

In order to be eligible for the study, subjects with transtibial amputation were required to be unilateral, without pain or ulcer on the residual limb, and with a residual-limb length not less than $13 \mathrm{~cm}$. Furthermore, they could not have volume fluctuation in the residual limb, could not depend on assistive devices such as a cane or crutches for ambulation, and had to have good upper-limb strength. 


\section{Procedures}

Two transtibial prostheses (Figure 1) were manufactured for each subject. Two different suspension systems were used: Seal-In X5 liner with valve (Icelock Expulsion Valve 551, Össur) and Dermo liner with shuttle lock (Icelock Clutch 4H 214, Össur).

Figure 1. Transtibial suspension systems: (a) Seal-In X5 liner (Össur; Reykjavik, Iceland) with transparent socket and valve and (b) Dermo liner (Össur) with transparent socket and shuttle lock. All prosthetic feet were Flex-Foot Talux (Össur) [16,18]. One of the researchers (registered prosthetist) designed, fit, and aligned all the prosthetic limbs. Two separate total surface bearing sockets were fabricated individually for each of the two liners that were used in the study. Transparent thermoplastic material (NorthPlex 12 mm, North Sea Plastics Ltd; Glasgow, United Kingdom) enabled us to check the socket fit. The subjects attended a gait training session in the Brace and Limb Laboratory (Department of Biomedical Engineering, University of Malaya, Malaysia).

The prosthetist ensured that there was no gait abnormality and that the fit of the prosthetic sockets was satisfactory.

We determined prosthetic alignment through bench, static (standing in an upright position), and 


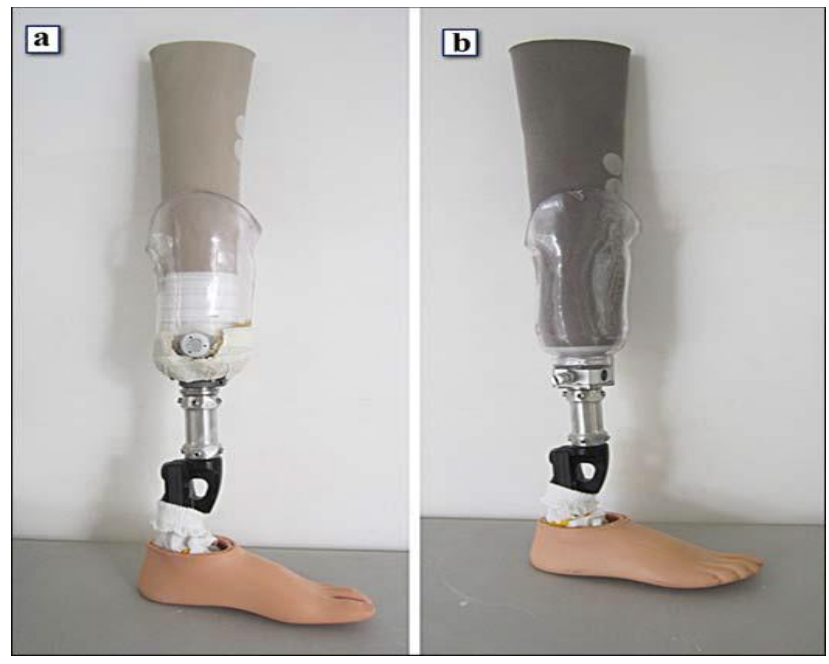

Figure 1.

Transtibial suspension systems: (a) Seal-In X5 liner (Össur; Reykjavik, Iceland) with transparent socket and valve and (b) Dermo liner (Össur) with transparent socket and shuttle lock.

dynamic (during walking) alignment. All subjects had an acclimation period of 4 weeks for each prosthetic device. To ensure subject safety, one definite socket was also made for each liner type for the 4-week acclimation period. Check sockets were used only during the kinematic experiments.

Following the trial period, we performed pistoning evaluation in the motion analysis laboratory with the Vicon 612 system using seven MXF20 motion capture cameras (Vicon; Los Angeles, California), which is believed to have an accuracy level of less than $\pm 0.1 \mathrm{~mm}$ [28]. We adopted a sampling rate of $200 \mathrm{~Hz}$ for the data collection. The signals from the motion analysis system were filtered by a Butterworth filter (cutoff frequency of $10 \mathrm{~Hz}$ ).

We fixed 16 reflective markers to the subjects' lower limbs in accordance with the Helen Hayes marker set. The knee and tibia markers for the prosthetic leg were located on the lateral proximal socket wall and the lateral distal end of the socket, respectively (Figure 2). We placed two additional markers on the liner under the knee 
joint level (LLin1) and $5 \mathrm{~cm}$ below that (LLin2) [16].

Because knee joint movement could affect the actual pistoning values, we positioned the additional markers (LLin1 and LLin2), aligned by laser liner, on the liner below the knee joint. With the transparent socket, the markers were visible through the hard socket and detectable by the cameras [16]. By fixing the markers to one segment (the shank), we avoided knee movements leading to unreal displacement.

The transparent socket could create some reflections that could be mistakenly considered as markers, therefore we used paper tape (except for the areas where additional markers were located) to mask the socket wall [16]. Prior to the test, we asked subjects to walk in the motion analysis laboratory in order to accustom themselves to the environment. Afterward, the subjects walked at a self-selected speed on an $8 \mathrm{~m}$ walkway. We recorded five successful trials per subject with each type of liner. We considered a trial to be successful if the cameras could capture all the markers. We could measure the pistoning by analyzing the markers' positions; however, in order to detect one gait cycle in each trial, we also used two Kistler force plates. There was a $1 \mathrm{~min}$ rest interval between the trials. We used the distance between the markers on the liner and on the socket to identify the piston motion.

The reproducibility of measurements was evaluated by intraobserver intrasession, intraobserver intersession, and interobserver intersession variabilities. Two observers 


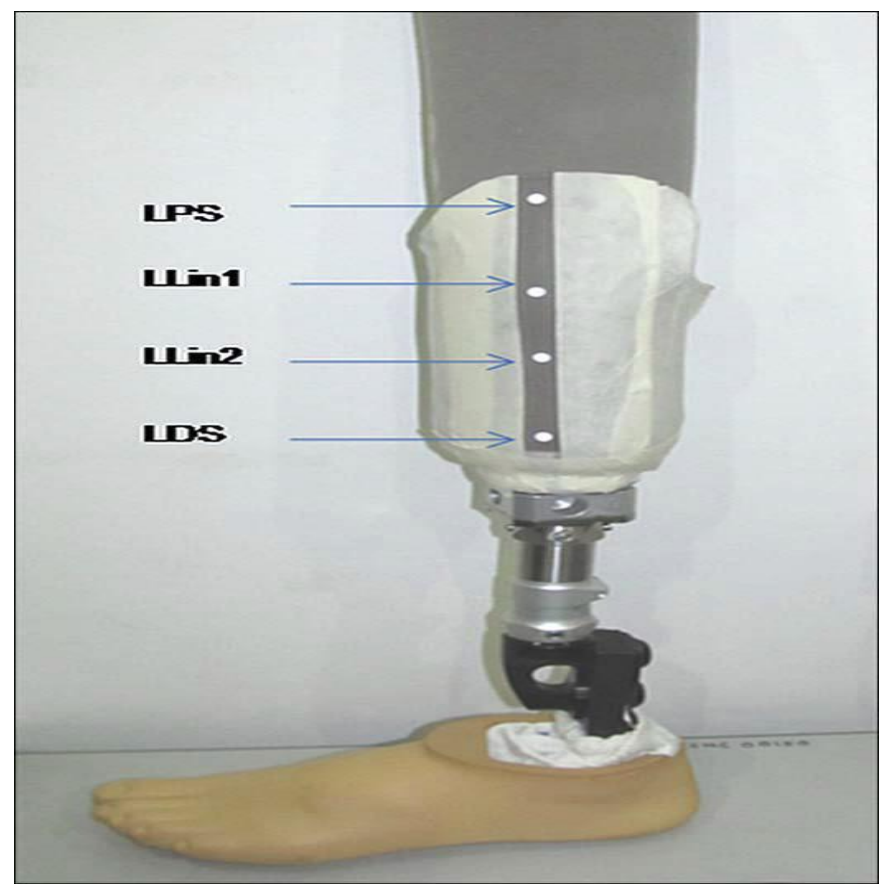

performed the experiments

Figure 2.

Marker positions on socket (lateral proximal socket wall [LPS]

and lateral distal end of socket [LDS]) and liner (LLin1 and LLin2).

Full text is available at :

http://www.ncbi.nlm.nih.gov/pubmed/23408214

http://eds.b.ebscohost.com/ehost/detail?sid=14da0d52-3122-463e-b2a9-

9d1ebd3b7aeb\%40sessionmgr115\&vid=1\&hid=110\&bdata=JnNpdGU9ZWhvc3QtbG|2ZQ\%3d\%3d 